ARTICLE

Received 10 Dec 2014 | Accepted 9 Jun 2015 | Published 23 Jul 2015 DOl: 10.1038/ncomms8777 OPEN

\title{
Observation of universal strong orbital-dependent correlation effects in iron chalcogenides
}

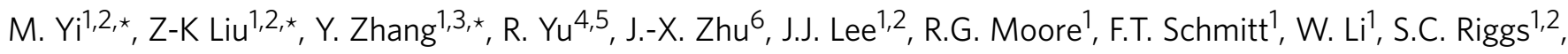

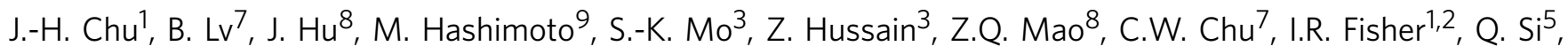
Z.-X. Shen ${ }^{1,2} \&$ D.H. Lu 9

Establishing the appropriate theoretical framework for unconventional superconductivity in the iron-based materials requires correct understanding of both the electron correlation strength and the role of Fermi surfaces. This fundamental issue becomes especially relevant with the discovery of the iron chalcogenide superconductors. Here, we use angle-resolved photoemission spectroscopy to measure three representative iron chalcogenides, $\mathrm{FeTe}_{0.56} \mathrm{Se}_{0.44}$, monolayer FeSe grown on $\mathrm{SrTiO}_{3}$ and $\mathrm{K}_{0.76} \mathrm{Fe}_{1.72} \mathrm{Se}_{2}$. We show that these superconductors are all strongly correlated, with an orbital-selective strong renormalization in the $d_{x y}$ bands despite having drastically different Fermi surface topologies. Furthermore, raising temperature brings all three compounds from a metallic state to a phase where the $d_{x y}$ orbital loses all spectral weight while other orbitals remain itinerant. These observations establish that iron chalcogenides display universal orbital-selective strong correlations that are insensitive to the Fermi surface topology, and are close to an orbital-selective Mott phase, hence placing strong constraints for theoretical understanding of iron-based superconductors.

\footnotetext{
${ }^{1}$ Stanford Institute for Materials and Energy Sciences, SLAC National Accelerator Laboratory and Stanford University, Menlo Park, California 94025, USA. 2 Departments of Physics and Applied Physics, and Geballe Laboratory for Advanced Materials, Stanford University, Stanford, California 94305 , USA.

${ }^{3}$ Advanced Light Source, Lawrence Berkeley National Lab, Berkeley, California 94720, USA. ${ }^{4}$ Department of Physics, Renmin University of China, Beijing 100872, China. ${ }^{5}$ Department of Physics and Astronomy, Rice University, Houston, Texas 77005, USA. ${ }^{6}$ Theoretical Division, Los Alamos National Laboratory, Los Alamos, New Mexico 87545, USA. ${ }^{7}$ Department of Physics, Texas Center for Superconductivity, University of Houston, Houston, Texas 77204, USA. ${ }^{8}$ Department of Physics and Engineering Physics, Tulane University, New Orleans, Louisiana 70118, USA. ${ }^{9}$ Stanford Synchrotron Radiation Lightsource, SLAC National Accelerator Laboratory, Menlo Park, California 94025, USA. * These authors contributed equally to this work. Correspondence and requests for materials should be addressed to Z.-X.S. (email: zxshen@stanford.edu) or to D.H.L. (email: dhlu@slac.stanford.edu).
} 
S ince the discovery of high-temperature superconductivity in the iron pnictides (FePns), extensive research efforts have revealed many common properties of these materials. In the vast material base of the FePns, all parent phases are metallic, and the observed electronic structures are largely consistent with the prediction of $a b$ initio local-density approximation (LDA) calculations $s^{1,2}$. These properties have led to theoretical understanding that the electron correlations in FePns are much weaker than in cuprate superconductors, whose parent phases are Mott insulators. Furthermore, the observation of comparable sized hole pockets at the Brillouin zone (BZ) centre and electron pockets at the $\mathrm{BZ}$ corner have resulted in the proposal that such a Fermi surface (FS) topology is ubiquitous and essential to superconductivity in FePns, and pairing in the FePns is mediated by antiferromagnetic fluctuations via FS nesting between the hole and electron Fermi pockets ${ }^{3}$. On the other hand, there have also been theoretical proposals of 'incipient Mott localization' for which the system is metallic but on the verge of localization ${ }^{4-6}$, supported by the normal state bad metal behaviour from optical conductivity measurements ${ }^{7}$ and the large fluctuating magnetic moment comparable to the antiferromagnetic insulating copper oxides ${ }^{8}$. This discussion became especially relevant with the more recent discovery of iron chalcogenides $(\mathrm{FeChs})^{9-11}$, which not only possess large local magnetic moments ${ }^{12}$ and insulating phases ${ }^{13}$, but also include compounds that lack hole pockets needed for FS nesting yet have comparable superconducting transition temperatures $\left(T_{\mathrm{C}} \mathrm{s}\right)$ as FePns ${ }^{14-17}$

There are currently three major classes of $\mathrm{FeCh}$ superconductors: $\mathrm{FeTe}_{1-x} \mathrm{Se}_{x}, \mathrm{~K}_{x} \mathrm{Fe}_{2}{ }_{y} \mathrm{Se}_{2}$, and FeSe film grown on $\mathrm{SrTiO}_{3}$. In the $\mathrm{FeTe}_{1-x} \mathrm{Se}_{x}$ family, superconductivity is achieved with isovalent substitution of Se for Te that suppresses magnetic order in the FeTe end ${ }^{18}$, and becomes optimal in $\mathrm{FeTe}_{0.56} \mathrm{Se}_{0.44}$ (FTS), where $T_{\mathrm{C}}$ is $14.5 \mathrm{~K}$. The $\mathrm{K}_{x} \mathrm{Fe}_{2}-y \mathrm{Se}_{2}$ family has in its phase diagram insulating phases with magnetic moments as large as $3.3 \mu_{\mathrm{B}}$ per $\mathrm{Fe}^{10,12,13}$, and optimal $T_{\mathrm{C}}$ is $32 \mathrm{~K}$, such as achieved in $\mathrm{K}_{0.76} \mathrm{Fe}_{1.72} \mathrm{Se}_{2}$ (KFS). FeSe film grown on $\mathrm{SrTiO}_{3}$ is the latest addition to the $\mathrm{FeCh}$ family, with monolayer film (FS/STO) having a record $T_{\mathrm{C}}$ possibly exceeding $65 \mathrm{~K}$ (refs $11,16,17$ ). In a previous angle-resolved photoemission spectroscopy (ARPES) study ${ }^{19}$ on KFS, we found the low-temperature state to be a metallic state with orbital-dependent renormalizationwhere the $d_{x y}$ orbital-dominated bands are strongly renormalized as compared with other orbitals. Raising temperature drives the material to an orbital-selective Mott phase (OSMP) in which the $d_{x y}$ orbital completely loses spectral weight while other orbitals remain itinerant. Subsequently, such a temperature scale was also identified by terahertz spectroscopy ${ }^{20}$, Hall measurements ${ }^{21}$ and pump-probe spectroscopy ${ }^{22}$, where the slight temperature scale variations are due to the different definitions used. This motivates the usage of orbital selectivity to address the fundamental open question of whether it is the electron correlation strength or the nature of the FS that plays a predominant role in the microscopic physics of the $\mathrm{FeCh}$ materials.

In this work, we study systematically the optimal superconducting members of the three FeCh families, FTS, KFS and FS/STO, using ARPES. We show that, in the low-temperature state, in contrast to the FePns, all of the FeChs are in a strongly correlated regime where strong orbital-selective renormalization is observed on the $d_{x y}$ bands, despite having drastically different FS topologies. In addition, by increasing temperature, all of the FeChs crossover into a phase where the $d_{x y}$ orbital completely loses spectral weight while other orbitals remain metallic. These observations showcase the universally strong orbital-selective electron correlations in the FeChs, and that the superconductivity in the FeChs, which is insensitive to FS topology, occurs in proximity to an orbital-selective Mott phase, placing strong constraints on the theoretical understanding of the iron-based superconductors.

\section{Results}

Orbital-selective band renormalization at low temperatures. The generic electronic structure of iron-based superconductors (FeSCs) consists of three hole bands at the BZ centre, $\Gamma$, and two electron bands at the $\mathrm{BZ}$ corner, $M$. The hole bands are predominantly of $d_{x z}, d_{y z}$ and $d_{x y}$ orbital characters, while the electron bands are $d_{x z}$ and $d_{x y}$ along $\Gamma-M$. The relative positions of these bands with respect to each other as well as to the Fermi level $\left(E_{\mathrm{F}}\right)$ could vary with differences in lattice parameters and doping level. Hence, the FS topology among different FeSCs could be qualitatively different, as shown in Fig. 1a-d, where the Fermi pockets at the BZ centre vary from being hole-like to non-existent to electron like. The measured band structure along the $\Gamma-M$ high-symmetry direction for the three compounds are shown in Fig. 1e-g, in comparison to that for the optimally Co-doped $\mathrm{BaFe}_{2} \mathrm{As}_{2}$ (BFCA) (Fig. 1h), an FePn as a reference. For FTS (Fig. 1i), one of the hole bands crosses $E_{\mathrm{F}}$, and both electron bands cross $E_{\mathrm{F}}$ at $M$, resulting in roughly compensated hole pocket at $\Gamma$ and electron pockets at $M$ (Fig. 1a), consistent with isovalent substitution for this compound. For both FS/STO (Fig. 1j) and KFS (Fig. 1k) in contrast, only the electron bands cross $E_{\mathrm{F}}$ while the hole band tops are well below $E_{\mathrm{F}}$, with an additional small electron pocket at $\Gamma$ in KFS. Thus, there is heavy electron doping in both compounds as reflected in a FS topology consisting only of electron pockets (Fig. 1b,c). Comparing the band structure of the three FeChs to the FePns, we notice a significant difference near the $M$ point-there is an apparent gap between the bottom of the electron bands and the top of the hole band in all three FeChs, in sharp contrast to BFCA (Fig. 11), in which the $d_{x z}$ electron band bottom is degenerate with the $d_{y z}$ hole band top.

Generally in FeSC, this degeneracy between the $d_{x z}$ electron band bottom and $d_{y z}$ hole band top at the zone corner is protected by the $\mathrm{C}_{4}$ rotational symmetry, as seen in BFCA (Fig. 11) and corresponding LDA calculations (Fig. 2a). This degeneracy is only lifted with the breaking of $\mathrm{C}_{4}$ symmetry, as in the orthorhombic phase of underdoped $\mathrm{BFCA}^{23}, \mathrm{NaFeAs}^{24,25}$, bulk $\mathrm{FeSe}^{26-29}$ and multilayer $\mathrm{FeSe}$ film ${ }^{30,31}$, where a splitting between the corresponding $d_{x z}$ bands and $d_{y z}$ bands occurs, in addition to a doubling of the bands from twinning effects due to the orthorhombic distortion. However, no static $\mathrm{C}_{4}$ symmetry breaking has been reported for any of the FeChs studied here, nor are twinning effects observed here that is expected from a broken symmetry due to orthorhombic distortion. Rather, this apparent gap can be explained by a strong orbital-dependent band renormalization and band hybridization. As the schematic shown in Fig. 2, the LDA-calculated $d_{x y}$ electron band bottom is deeper than that of the $d_{x z}$ band. If the $d_{x y}$ orbital is strongly renormalized compared with the other orbitals, the $d_{x y}$ electron band bottom, that is, the corresponding $d_{x y}$ hole band top, would rise above that of the $d_{x z}$ electron band (Fig. 2b). The heavily renormalized $d_{x y}$ hole band then crosses the $d_{x z}$ electron band and the two bands hybridize such that a gap appears at the $M$ point without lifting the $d_{x z} / d_{y z}$ degeneracy protected by $\mathrm{C}_{4}$ symmetry (Fig. 2c). Evidence for two nearly degenerate electron bands can be seen in the high-resolution spectra acquired on FS/STO (Fig. 3a). As an aside, we note that in the unrenormalized case (Fig. 2a), a hybridization gap between the $d_{x y}$ electron band and $d_{y z}$ hole band is not observed. This is because when considering the hopping via the chalcogen atoms along this high-symmetry direction $(x)$, both $d_{x y}$ and $d_{x z}$ are odd while $\mathrm{d}_{y z}$ is even. Hence $d_{x y}$ does not mix with $d_{y z}$ to produce a hybridization gap ${ }^{32}$. 
a
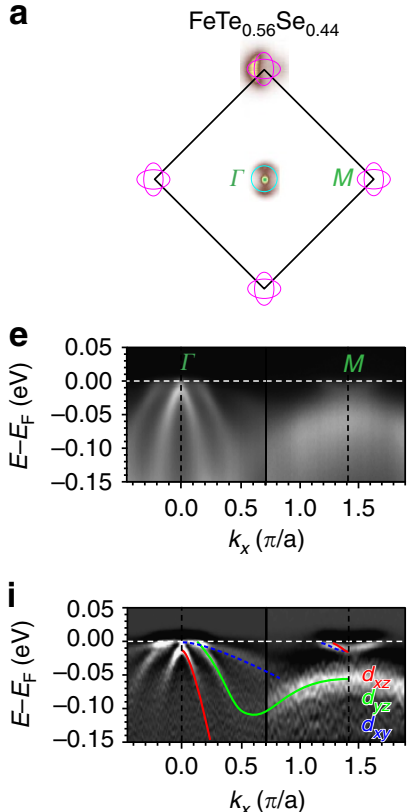

b


C
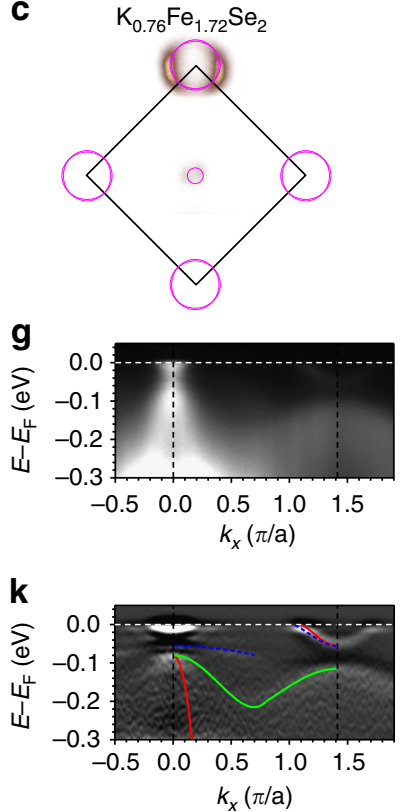

d


Figure 1 | Low-temperature band structure of iron chalcogenides in comparison to iron pnictide. Fermi surfaces measured on (a) FeTe ${ }_{0.56} \mathrm{Se}_{0.44}$ (FTS), (b) monolayer FeSe film on $\mathrm{SrTiO}_{3}$ (FS/STO), (c) $\mathrm{K}_{0.76} \mathrm{Fe}_{1.72} \mathrm{Se}_{2}$ (KFS) and (d) $\mathrm{Ba}\left(\mathrm{Fe}_{0.93} \mathrm{Co}_{0.07}\right)_{2} \mathrm{As}_{2}$ (BFCA), shown in $\mathrm{BZ}$ notation corresponding to 2-Fe unit cell (For comparison purposes, we use the $M$ point to denote the BZ corner where the electron pockets live for all compounds and LDA, even though for 122 crystal structures, this is the $X$ point.), with schematic outlines shown in cyan (magenta) for hole (electron) Fermi pockets. (e) Spectral image of FTS along the $\Gamma-M$ high-symmetry direction, taken with $22 \mathrm{eV}(26 \mathrm{eV})$ photons for near the $\Gamma(M)$ point. Measurements along the same cut for (f) FS/STO, (g) KFS and (h) BFCA, with photon energies of 22, 26 and $47.5 \mathrm{eV}$, respectively. In-plane polarization was odd with respect to the cut for all measurements, (e-g) has additional out-of-plane polarization. (i-I) Second energy derivatives for the spectral images above. Observable bands are marked with dominant orbital character (red: $d_{x z}$ green: $d_{y z}$ and blue: $d_{x y}$ ).
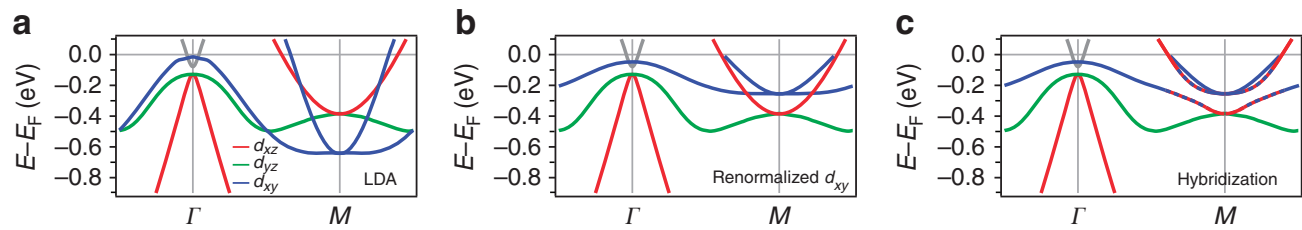

Figure 2 | Schematics of the effect of orbital-dependent band renormalizations. (a) LDA calculations for KFS ${ }^{41}$. (b) Schematic based on a with $d_{x y}$ orbital strongly renormalized. (c) Schematic based on $\mathbf{b}$ by introducing hybridization between $d_{x y}$ band and $d_{x z}$ electron band.

This interpretation is further supported by the observed strong renormalization of the $d_{x y}$ hole band near $\Gamma$, which is significantly more renormalized than the $d_{x z} / d_{y z}$ hole bands, clearly seen in all three compounds (Fig. 1i-k). For FTS, the $d_{x y}$ hole band is strongly renormalized by a factor of $\sim 16$ compared with LDA calculations, while $d_{x z}$ and $d_{y z}$ bands are only renormalized by factors of $\sim 4$. Moreover, in the $\mathrm{FeTe}_{1-x} \mathrm{Se}_{x}$ family, it has been found that the $d_{x y}$ band renormalization factor strongly increases towards the FeTe end compared with that of $d_{x z} / d_{y z}$, further revealing the strong orbital dependence in the itinerant to localized crossover in this system ${ }^{33}$. For KFS, the $d_{x y}$ hole band is renormalized by a factor of $\sim 10$ compared with the factor of $\sim 3$ for $d_{x z} / d_{y z}$ bands ${ }^{19}$. This is even more apparent in FS/STO, where enhanced $d_{x y}$ orbital matrix elements in the second BZ shows the nearly flat $d_{x y}$ hole band extending towards the bottom of the shallow electron bands at $M$ with a noticeable hybridization gap (Fig. 3b). In contrast, in BFCA, the renormalization factor for $d_{x y}$ hole band is comparable to that of the $d_{x z} / d_{y z}$ hole bands $(2 \sim 3)$ (ref. 2), as can be seen in the band slopes in the second derivative plot (Fig. 11). In summary, all three FeCh systems show much stronger renormalization in the $d_{x y}$ orbital compared with the $d_{x z} /$ $d_{y z}$ orbitals in the low-temperature state, in contrast to the FePns. Here we would like to note that while ARPES is a surfacesensitive probe, the universal orbital-selective renormalization among the FeChs despite their different structure and surface terminations indicate that these properties must represent the bulk, rather than a result of extrinsic variation of surface properties.

Temperature dependence. Next, by raising temperature sufficiently high, we notice that in all three FeCh systems, the $d_{x y}$ orbital-dominated bands lose spectral weight completely (Fig. 4), as reported previously for $\mathrm{KFS}^{19}$. This can be seen first in the disappearance of the shallow $d_{x y}$ hole band near the $\Gamma$ point. In the low-temperature state of FTS, the $d_{x y}$ hole band crosses the $d_{y z}$ hole band near $\Gamma$, and a small hybridization gap appears as can be seen in the discontinuous intensity pattern of the strong $d_{y z}$ hole band (Fig. 4a). At high temperature, this discontinuity disappears as only the $d_{x z}$ and $d_{y z}$ hole bands remain (Fig. $4 \mathrm{~d}$ ). In KFS, the nearly flat $d_{x y}$ hole band in the low-temperature state is entirely above the $d_{x z} / d_{y z}$ hole band tops (Fig. 4c), and clearly disappears 
at high temperatures (Fig. 4f). The second evidence for the disappearance of $d_{x y}$ orbital at high temperatures is the vanishing of the apparent gap between the electron band bottom and the $d_{y z}$ hole band top at $M$. As shown in the schematic (Fig. $4 \mathrm{~g}, \mathrm{~h}$ ), when the $d_{x y}$ band disappears, its hybridization gap with the $d_{x z}$ electron band naturally vanishes, recovering the deeper non-hybridized $d_{x z}$ electron band whose bottom is degenerate with the $d_{y z}$ hole band top as expected. This is clearly seen in the high-temperature data in all three systems (Fig. 4d-f). Here we note that while KFS is known to have phase separation issues with the existence of superconducting regions and insulating regions ${ }^{19}$, FTS and FS/ STO, on the other hand, do not have such complication. Hence the universal behaviour of vanishing $d_{x y}$ spectral weight with raised temperature observed here reflects an intrinsic effect rather than a result of intricate phase separation in KFS.

To examine this temperature dependence more carefully, we have quantitatively analysed the spectral weight of each FeCh system. For FTS, we track the $d_{x y}$ hole band slightly away from the $\Gamma$ point where it is well separated from the $d_{y z}$ hole band (Fig. 5a). From the energy distribution curve at this momentum, we fit Gaussian peaks for both $d_{x y}$ hole band near $E_{\mathrm{F}}$ and $d_{y z}$ hole band at higher energies, along with a Shirley background (Fig. 5d), and track the integrated spectral weight of the $d_{x y}$ and $d_{y z}$ peaks as a function of temperature. Comparing these two orbitals, we see that the $d_{x y}$ spectral weight drops to zero $\sim 110 \mathrm{~K}$, in contrast to a very weak decrease of the $d_{y z}$ spectral weight.
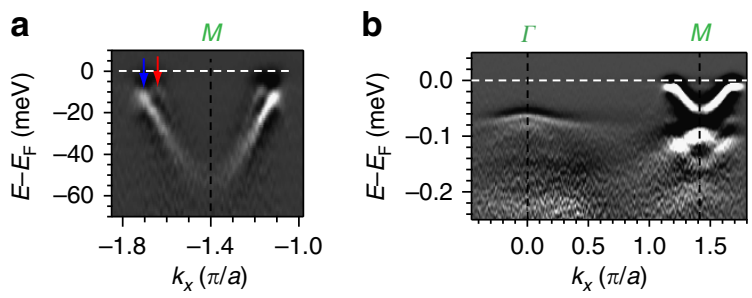

Figure 3 | Selected spectra from FS/STO. (a) High-resolution spectra of FS/STO showing the presence of two electron bands around $M$. (b) $\Gamma-M$ high-symmetry cut of FS/STO taken in the second BZ, where $d_{x y}$ orbital matrix elements are strong. The $d_{x y}$ hole band has a lower band top at $\Gamma$ than $d_{x z} / d_{y z}$, hence it crosses and hybridizes with the $d_{x z} / d_{y z}$ hole bands, resulting in the apparent sharper curvature near $\Gamma$.
This is very similar to the situation in FS/STO and KFS, where we track the spectral weight of the $d_{x y}$ orbital at the $d_{x y}$ electron band bottom at $M$. For KFS, the fitted peak area precipitously drops $\sim 100 \mathrm{~K}$, and approaches zero above $180 \mathrm{~K}$ (Fig. 5i). For FS/ STO, the $d_{x y}$ spectral weight approaches zero above $150 \mathrm{~K}$ while that of the $d_{\mathrm{yz}}$ orbital remains finite (Fig. 5h), demonstrating the orbital dependence of this temperature evolution.

Theoretical calculations. For all three FeCh superconductors studied, two observations are universal: (i) strong orbital-dependent renormalization at low temperatures and (ii) disappearance of $d_{x y}$ spectral weight with raised temperatures. Theoretically, these behaviours can be understood in proximity to an OSMP, as predicted by both a slave-spin mean-field method ${ }^{34}$ and dynamical mean-field theory method ${ }^{35}$ taking into account sufficiently strong intra-orbital Coulomb repulsion $U$ and Hund's coupling $J$. These works show that the FeChs are close to an OSMP in which the $d_{x y}$ orbital is completely Mott localized while the other orbitals remain itinerant, and that a temperature scale could be identified where the $d_{x y}$ orbital loses coherence and spectral weight, together with its hybridization to the other orbitals.

The orbital selectivity arises from two factors ${ }^{19,34}$. The crystal field splitting of the tetragonal lattice makes the $d_{x y}$ orbital energy level higher than that of $d_{x z} / d_{y z}$. Because the threshold interaction for the Mott transition is larger for degenerate orbitals than for non-degenerate orbitals ${ }^{36}$, it is easier to localize the $d_{x y}$ electrons. The tendency towards OSMP is further enhanced when the projected bandwidth is different ${ }^{37}$, as is the case here with the $d_{x y}$ band being narrower than the $d_{x z} / d_{y z}$ bands. When the material is sufficiently close to such an OSMP, the $d_{x y}$ mass renormalization is much stronger than that of other orbitals, as observed in the low-temperature state of the FeChs. For the system to be close to this OSMP, it must exhibit strong electron correlations, which from the two theoretical studies is shown to be a combined result of Coulomb interactions $U$ and Hund's coupling $J$.

Among the three FeChs, FTS is undoped, with $n=6$, while FS/STO and KFS are electron doped, with $n=6.12$ and 6.15 , as estimated from FS volume counting. Figure 6 shows our calculated transition temperatures into the OSMP versus $U$ phase diagrams for systematic dopings from $n=6-6.15$. The blue shading indicates the $d_{x y}$ quasiparticle spectral weight, $Z_{x y}$, for each doping. When $Z_{x y}$ drops to zero, the system enters the OSMP. This transition boundary is marked by solid blue lines in
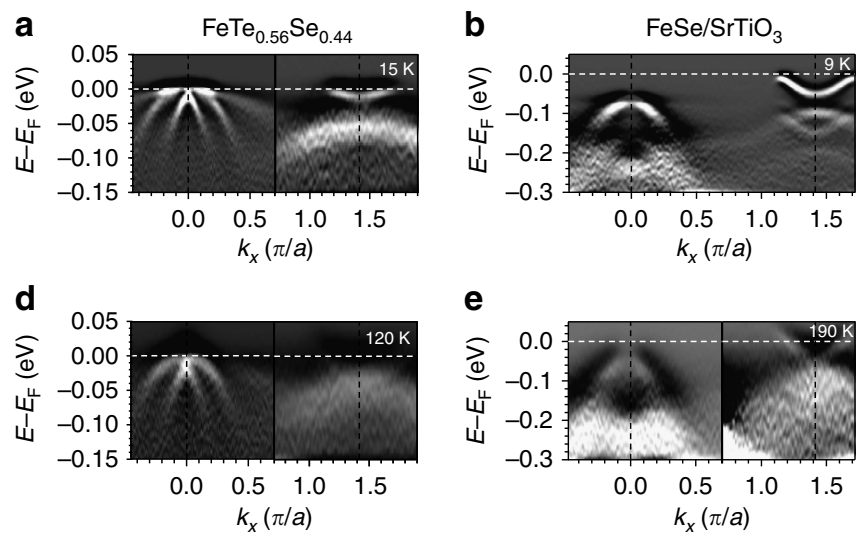

e

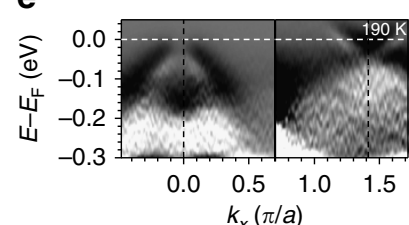

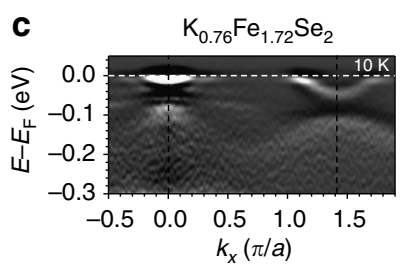

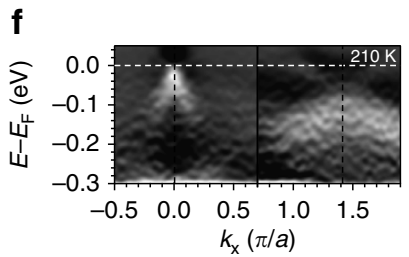

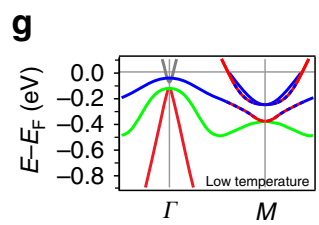

h

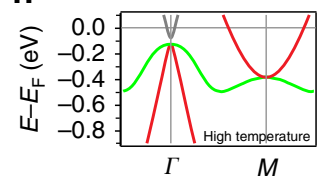

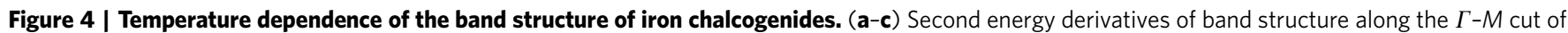
FTS, FS/STO and KFS, same as that of Fig. 1i-k, taken in the low-temperature state at 15, 9 and 10 K, respectively. (d-f) Same as that of a-c but taken in the high-temperature state at 120, 190 and $210 \mathrm{~K}$, respectively. (g) Schematic showing the band structure in the low-temperature state with finite spectral weight of $d_{x y}$ orbital (red: $d_{x z}$ green: $d_{y z}$ and blue: $d_{x y}$ ). (h) Schematic showing the band structure in the high-temperature state after $d_{x y}$ orbital completely loses spectral weight. 


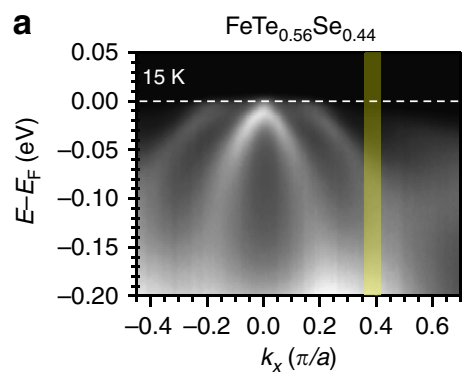

d

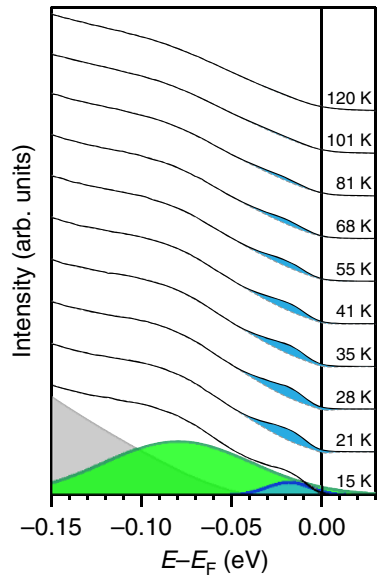

g

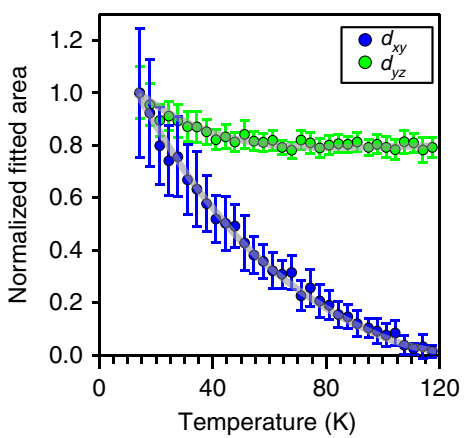

b

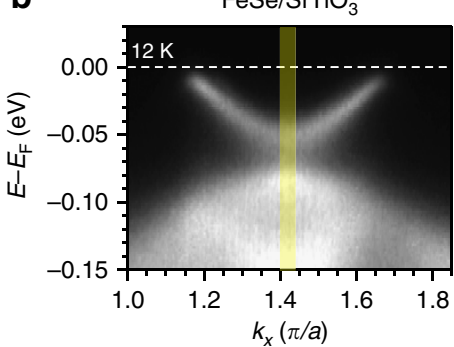

e

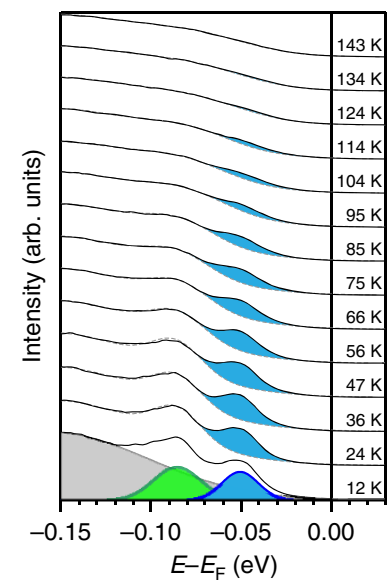

h

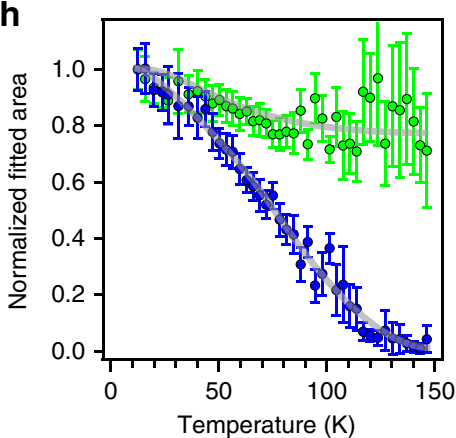

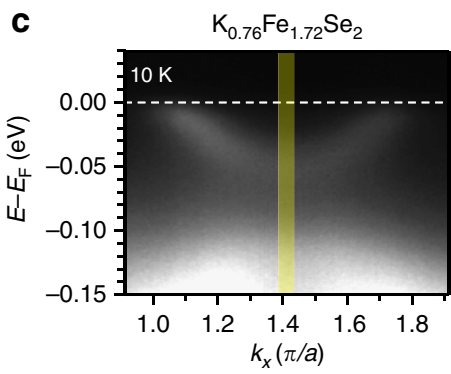

f

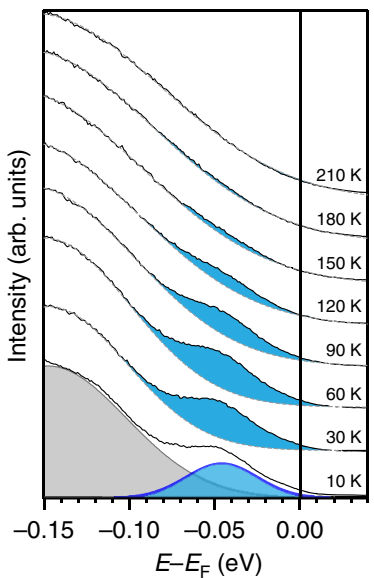

i

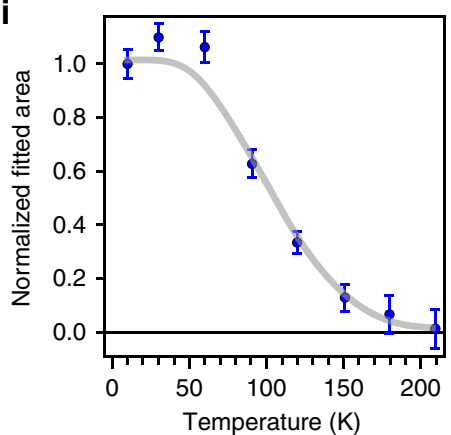

Figure 5 | Quantitative analysis of temperature evolution in the iron chalcogenides. (a-c) Raw spectral images of FTS, FS/STO and KFS taken in the lowtemperature state. Yellow regions mark the momentum ranges integrated for energy distribution curve (EDC) analysis for each compound. (d) Integrated EDCs in the yellow region of a for FTS at selected temperatures, fitted by a Shirley background (grey), a Gaussian for the $d_{x y}$ band (blue), and a Gaussian for the $d_{y z}$ band (green), convolved by the Fermi-Dirac function. (e) Integrated EDCs in the yellow region of $\mathbf{b}$ for FS/STO at selected temperatures, with a Gaussian background (grey), a Gaussian for the $d_{x y}$ band (blue) and a Gaussian for the $d_{y z}$ band (green). (f) Integrated EDCs in the yellow region of $\mathbf{c}$ for KFS at selected temperatures, fitted by a Gaussian background (grey), and a Gaussian for the $d_{x y}$ band (blue). Residual spectral weight for the $d_{x y}$ peak is shaded for each temperature for all compounds. Fitted peaks for the lowest temperature are shown for each compound. (g-i) Temperature dependence of the fitted areas of the $d_{x y}$ and $d_{y z}$ peaks for FTS, FS/STO and KFS. Guides to eye are drawn in grey to show the trends. All curves are normalized by the initial value of the peak area. The error bars in $\mathbf{g}-\mathbf{i}$ are error bars resulted from the fitting.

Fig. 6. Furthermore, we see that for and only for integer filling, $n=6$ in the calculated doping range, there exists a Mott insulator phase (marked by a red line), where all orbitals become Mott localized with sufficiently large $U$. With increasing electron filling, the critical $U$ for the system entering the OSMP at a given temperature shifts to larger values, as can be seen by tracing the blue lines at both 10 and $240 \mathrm{~K}$, indicating that electron doping brings the system away from the OSMP. This is consistent with the observation that the temperature at which the $d_{x y}$ spectral weight disappears increases from FTS $(110 \mathrm{~K})$ to the doped FS/STO (150 K) and KFS (180 K). Here we note that the resistivity for the $\mathrm{FeTe}_{1-} \mathrm{Se}_{x}$ family exhibits a weak hump ${ }^{18}$ suggestive of a crossover from insulating-like to metallic-like behaviour much like the case of $\mathrm{KFS}^{10}$, and this crossover temperature scale shifts to lower value with decreasing $x$, consistent with the stronger renormalization values towards the $\mathrm{FeTe}$ end $^{33}$ and the understanding that the low-temperature state of FeTe end sits closer to the OSMP.

\section{Discussion}

The strong orbital-selective behaviour in the FeCh family is a manifestation of its strong electron correlations, and suggests that they may serve as a bridge between the strongly correlated cuprates as doped Mott insulators and the weaker correlated FePns that are more itinerant. The OSMP of the FeChs can only occur when the overall electron correlations are strong. In that sense, the existence of an OSMP indicates the presence of strong 


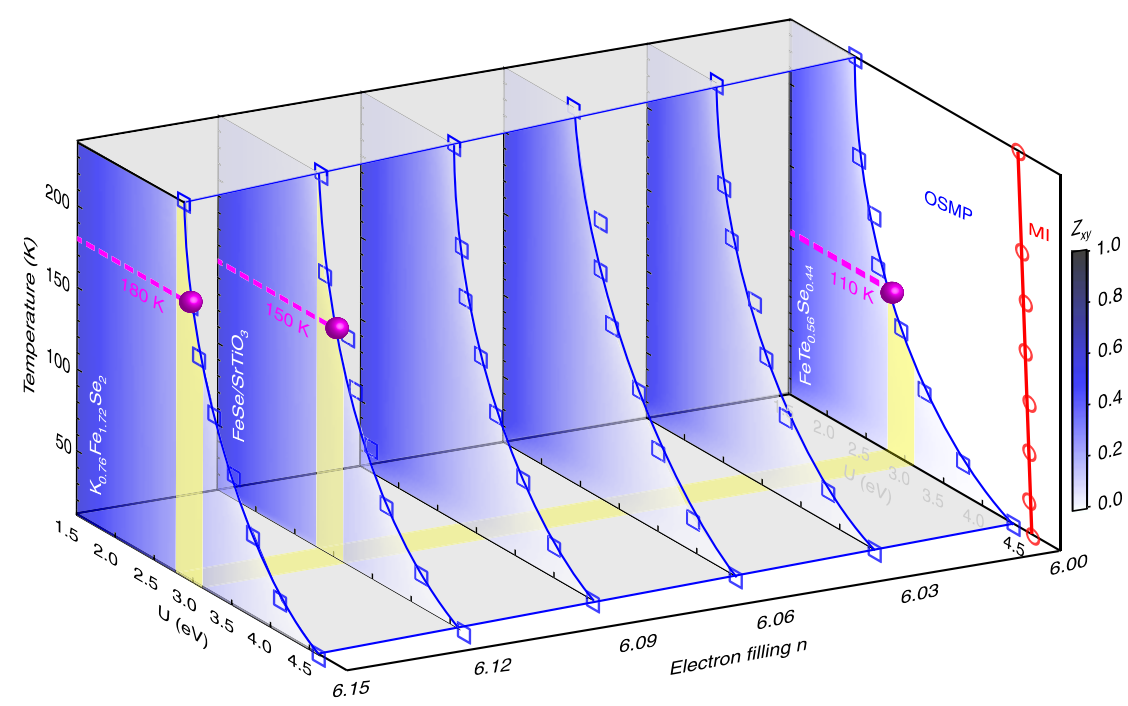

Figure 6 | Calculated phase diagram of the OSMP as a function of temperature, $\boldsymbol{U}$, and electron filling. Slave-spin mean-field phase diagrams of the five-orbital Hubbard model at systematic electron fillings from $n=6-6.15$. See Methods for calculation details. Blue shading shows the $d_{x y}$ quasiparticle spectral weight, $Z_{x y}$. The OSMP phase boundary is shown by solid blue lines connecting blue squares marking the calculated temperatures where $Z_{x y}$ drops to zero. A Mott insulator (MI) phase exists for $n=6$, where all orbitals have zero spectral weight. Its phase boundary is marked by a red line. The temperatures at which $Z_{x y}$ is observed to vanish in FTS, FS/STO and KFS are marked by magenta dotted lines. From these temperatures, the critical U's for FTS, FS/STO and KFS can be estimated, which is shown as a yellow guide to the eye strip at fixed U. Hund's $J$ is fixed at $0.6 \mathrm{eV}$. Here, electron doping bring the system away from the OSMP, as seen in the increasing critical $U$ at fixed temperature (blue lines at 10 and $240 \mathrm{~K}$ ) as well as the increasing critical temperature at fixed $U$ (magenta dots).

correlations, such that the system is in proximity to a Mott transition. The latter links these materials to the cuprates, where optimal superconductivity develops not too far from a Mott insulating state. The linkage can be made more explicit by the Hund's coupling-induced suppression of the interorbital coupling in the case of the iron-based materials ${ }^{19,34,38}$. This is relevant to the current discussion on the pairing mechanism of FeSC in both KFS and FS/STO as the lack of hole pockets do not seem to prevent them from superconducting at temperatures comparable to or even higher than FePns, which is unexpected from a weakcoupling FS nesting picture ${ }^{3}$. In a recent theoretical study ${ }^{39}$, it has been shown that under a strong coupling approach, where the driving force for pairing comes from the close-neighbour exchange interactions, the pairing strength can be comparable in the FeChs and FePns, as they approach a Mott transition by increasing exchange interaction in the former and reducing the renormalized bandwidth in the latter. The observed universal strong correlation in the FeChs and proximity to an OSMP here supports such a scenario.

\section{Methods}

Sample growth. High-quality single crystals of $\mathrm{K}_{0.76} \mathrm{Fe}_{1.72} \mathrm{Se}_{2}$ and $\mathrm{FeTe}_{0.56} \mathrm{Se}_{0.44}$ were grown using the flux method ${ }^{19,40}$. Monolayer FeSe films were grown on $\mathrm{SrTiO}_{3}$ using molecular beam epitaxy ${ }^{17}$.

ARPES measurements. ARPES measurements were carried out at beamline 5-4 of the Stanford Synchrotron Radiation Lightsource and beamline 10.0.1 of the Advanced Light Source using SCIENTA R4000 electron analyzers. The total energy resolution was set to $10 \mathrm{meV}$ and the angular resolution was $0.3^{\circ}$. Single crystals were cleaved in situ at $10 \mathrm{~K}$ for each measurement. The FeSe films were transported to the beamline under vacuum and further annealed before measurements. All measurements were done in ultra high vacuum with a base pressure lower than $4 \times 10^{-11}$ torr.

Theoretical calculations. The theoretical calculations were done using a slavespin mean-field method on a five-orbital Hubbard model ${ }^{34}$, with tight-binding parameters for FTS. Hund's $J$ is fixed at $0.6 \mathrm{eV}$.

\section{References}

1. Lu, D. H. et al. Electronic structure of the iron-based superconductor LaOFeP. Nature 455, 81-84 (2008).

2. Yi, M. et al. Electronic structure of the $\mathrm{BaFe}_{2} \mathrm{As}_{2}$ family of iron-pnictide superconductors. Phys. Rev. B 80, 024515 (2009).

3. Mazin, I. I., Singh, D. J., Johannes, M. D. \& Du, M. H. Unconventional Superconductivity with a sign reversal in the order parameter of $\mathrm{LaFeAsO}_{1-x} \mathrm{~F}_{x}$. Phys. Rev. Lett. 101, 057003 (2008).

4. Si, Q. \& Abrahams, E. Strong correlations and magnetic frustration in the high $T_{\mathrm{C}}$ iron pnictides. Phys. Rev. Lett. 101, 076401 (2008).

5. Yu, R., Zhu, J.-X. \& Si, Q. Mott transition in modulated lattices and parent insulator of $(\mathrm{K}, \mathrm{Tl})_{y} \mathrm{Fe}_{x} \mathrm{Se}_{2}$ superconductors. Phys. Rev. Lett. 106, 186401 (2011).

6. Yin, Z. P., Haule, K. \& Kotliar, G. Kinetic frustration and the nature of the magnetic and paramagnetic states in iron pnictides and iron chalcogenides. Nat. Mater. 10, 932-935 (2011).

7. Qazilbash, M. M. et al. Electronic correlations in the iron pnictides. Nat. Phys 5, 647-650 (2009).

8. Liu, M. et al. Nature of magnetic excitations in superconducting $\mathrm{BaFe}_{1.9} \mathrm{Ni}_{0.1} \mathrm{As}_{2}$. Nat. Phys. 8, 376-381 (2012).

9. Hsu, F.-C. et al. Superconductivity in the PbO-type structure $\alpha$-FeSe. Proc. Natl Acad. Sci. 105, 14262-14264 (2008).

10. Guo, J. et al. Superconductivity in the iron selenide $\mathrm{K}_{\mathrm{x}} \mathrm{Fe}_{2} \mathrm{Se}_{2}(0 \leq \mathrm{x} \leq 1.0)$. Phys Rev. B 82, 180520 (2010).

11. Wang, Q. Y. et al. Interface induced high temperature superconductivity in single unit-cell FeSe films on $\mathrm{SrTiO}_{3}$. Chin. Phys. Lett. 29, 037402 (2012).

12. Bao, W. et al. A novel large moment antiferromagnetic order in $\mathrm{K}_{0.8} \mathrm{Fe}_{1.6} \mathrm{Se}_{2}$ superconductor. Chin. Phys. Lett. 28, 086104 (2011).

13. Fang, M.-H. et al. Fe-based superconductivity with $T_{\mathrm{C}}=31 \mathrm{~K}$ bordering an antiferromagnetic insulator in ( $\mathrm{Tl}, \mathrm{K}) \mathrm{Fe}_{x} \mathrm{Se}_{2}$. Europhys. Lett. 94, 27009 (2011).

14. Zhang, Y. et al. Nodeless superconducting gap in $\mathrm{A}_{x} \mathrm{Fe}_{2} \mathrm{Se}_{2}(\mathrm{~A}=\mathrm{K}, \mathrm{Cs})$ revealed by angle-resolved photoemission spectroscopy. Nat. Mater. 10, 273-277 (2011).

15. Qian, T. et al. Absence of a holelike fermi surface for the iron-based $\mathrm{K}_{0.8} \mathrm{Fe}_{1.7} \mathrm{Se}_{2}$ superconductor revealed by angle-resolved photoemission spectroscopy. Phys. Rev. Lett. 106, 187001 (2011).

16. Liu, D. et al. Electronic origin of high-temperature superconductivity in singlelayer FeSe superconductor. Nat. Commun. 3, 931 (2012).

17. Lee, J. J. et al. Interfacial mode-coupling as the origin of the enhancement of $T_{\mathrm{C}}$ in FeSe films on $\mathrm{SrTiO}_{3}$. Nature 515, 245-248 (2014).

18. Liu, T. J. et al. From $(\pi, 0)$ magnetic order to superconductivity with $(\pi, \pi)$ magnetic resonance in $\mathrm{Fe}_{1.02} \mathrm{Te}_{1-x} \mathrm{Se}_{x}$. Nat. Mater. 9, 718-720 (2010). 
19. Yi, M. et al. Observation of temperature-induced crossover to an orbitalselective Mott phase in $\mathrm{A}_{x} \mathrm{Fe}_{2}-\mathrm{Se}_{2}(\mathrm{~A}=\mathrm{K}, \mathrm{Rb})$ superconductors. Phys. Rev. Lett. 110, 067003 (2013).

20. Wang, Z. et al. Orbital-selective metal-insulator transition and gap formation above $T_{\mathrm{C}}$ in superconducting $\mathrm{Rb}_{1-x} \mathrm{Fe}_{2-y} \mathrm{Se}_{2}$. Nat. Commun. 5, 3202 (2014).

21. Ding, X. et al. Strong and nonmonotonic temperature dependence of Hall coefficient in superconducting $\mathrm{K}_{x} \mathrm{Fe}_{2-y} \mathrm{Se}_{2}$ single crystals. Phys. Rev. B 89, 224515 (2014).

22. Li, W. et al. Mott behaviour in $\mathrm{K}_{x} \mathrm{Fe}_{2}-{ }_{y} \mathrm{Se}_{2}$ superconductors studied by pumpprobe spectroscopy. Phys. Rev. B 89, 134515 (2014).

23. Yi, M. et al. Symmetry-breaking orbital anisotropy observed for detwinned $\mathrm{Ba}\left(\mathrm{Fe}_{1-x} \mathrm{Co}_{x}\right)_{2} \mathrm{As}_{2}$ above the spin density wave transition. Proc. Natl Acad. Sci. USA 108, 6878-6883 (2011).

24. $\mathrm{Yi}, \mathrm{M}$. et al. Electronic reconstruction through the structural and magnetic transition in detwinned NaFeAs. New J. Phys. 14, 073019 (2012).

25. Zhang, Y. et al. Symmetry breaking via orbital-dependent reconstruction of electronic structure in uniaxially strained NaFeAs. Phys. Rev. B 85, 085121 (2012).

26. Nakayama, K. et al. Reconstruction of band structure induced by electronic nematicity in FeSe superconductor. Phys. Rev. Lett. 113, 237001 (2014).

27. Shimojima, T. et al. Lifting of $\mathrm{xz} / \mathrm{yz}$ orbital degeneracy at the structural transition in detwinned FeSe. Phys. Rev. B 90, 121111(R) (2014).

28. Watson, M. D. et al. Emergence of the nematic electronic state in FeSe. Phys. Rev. B 91, 155106 (2015).

29. Zhang, P. et al.Evidence for intertwining orders in the electronic nematic state of FeSe. Preprint at http://arxiv.org/abs/1503.01390 (2015).

30. Tan, S. et al. Interface-induced superconductivity and strain-dependent spin density waves in $\mathrm{FeSe} / \mathrm{SrTiO}_{3}$ thin films. Nat. Mater. 12, 634-640 (2013).

31. Zhang, Y. et al. Distinctive momentum dependence of the band reconstruction in the nematic state of FeSe thin film. Preprint at http://arxiv.org/abs/ $1503.01556(2015)$

32. Lee, P. A. \& Wen, X.-G. Spin-triplet p-wave pairing in a three-orbital model for iron pnictide superconductors. Phys. Rev. B 78, 144517 (2008).

33. Liu, Z. K. et al. Experimental observation of incoherent-coherent crossover and orbital dependent band renormalization in iron chalcogenide superconductors. Preprint at http://arxiv.org/abs/1505.03074 (2015).

34. Yu, R. \& Si, Q. Orbital-selective Mott phase in multiorbital models for alkaline iron selenides $\mathrm{K}_{1-x} \mathrm{Fe}_{2-y} \mathrm{Se}_{2}$. Phys. Rev. Lett. 110, 146402 (2013).

35. Yin, Z. P., Haule, K. \& Kotliar, G. Fractional power-law behaviour and its origin in iron-chalcogneide and ruthenate superconductors: insights from first-principles calculations. Phys. Rev. B 86, 195141 (2012).

36. de' Medici, L., Hassan, S. R., Capone, M. \& Dai, X. Orbital-selective Mott transition out of band degeneracy lifting. Phys. Rev. Lett. 102, 126401 (2009).

37. Anisimov, V., Nekrasov, I. A., Kondakov, D. E., Rice, T. M. \& Sigrist, M. Orbital-selective Mott-insulator transition in $\mathrm{Ca}_{2}-{ }_{x} \mathrm{Sr}_{x} \mathrm{RuO}_{4}$. Eur. Phys. J. B 25, 191 (2002).

38. de' Medici, L., Giovannetti, G. \& Capone, M. Selective Mott physics as a key to iron superconductors. Phys. Rev. Lett. 112, 177001 (2014).
39. Yu, R., Goswami, P., Si, Q., Nikolic, P. \& Zhu, J.-X. Superconductivity at the border of electron localization and itinerancy. Nat. Commun. 4, 2783 (2013).

40. Liu, T. J. et al. Charge-carrier localization induced by excess Fe in the superconductor $\mathrm{Fe}_{1+y} \mathrm{Te}_{1-x} \mathrm{Se}_{x}$. Phys. Rev. B 80, 174509 (2009).

41. Nekrasov, I. A. \& Sadovskii, M. V. Electroni structure, topological phase transitions and superconductivity in $(\mathrm{K}, \mathrm{Cs})_{x} \mathrm{Fe}_{2} \mathrm{Se}_{2}$. JETP Lett. 93, 166-169 (2011).

\section{Acknowledgements}

ARPES experiments were performed at the Stanford Synchrotron Radiation Lightsource and the Advanced Light Source, which are both operated by the Office of Basic Energy Sciences, U.S. Department of Energy. The Stanford work is supported by the US DOE, Office of Basic Energy Science, Division of Materials Science and Engineering, under award number DE-AC02-76SF00515. The work at Rice is supported by NSF Grant DMR-1309531 and the Robert A. Welch Foundation Grant No. C-1411. The work at Renmin University is supported by the National Science Foundation of China Grant number 11374361, and the Fundamental Research Funds for the Central Universities and the Research Funds of Remnin University of China. The work at Tulane is supported by the NSF under grant DMR-1205469. The work at Los Alamos was supported by the U.S. DOE Office of Basic Energy Sciences.

\section{Author contributions}

M.Y., Z.K.L. and Y.Z. performed the ARPES experiments with assistance from M.H. and S.K.M. under the guidance of D.H.L. and Z.H. R.Y., J.X.Z. and Q.S. performed the theoretical calculations. J.J.L., R.G.M., F.T.S. and W.L. grew the FeSe films. S.C.R., J.H.C. and B.L. grew the KFS single crystals with guidance from I.R.F. and C.W.C. J.H. grew the FTS single crystals under the guidance of Z.Q.M. Z.X.S. and D.H.L. provided overall guidance and project coordination. The paper was written by M.Y., D.H.L., R.Y. and Q.S. with contributions from all authors.

\section{Additional information}

Competing financial interests: The authors declare no competing financial interests.

Reprints and permission information is available online at http://npg.nature.com/ reprintsandpermissions/

How to cite this article: Yi, M. et al. Observation of universal strong orbitaldependent correlation effects in iron chalcogenides. Nat. Commun. 6:7777 doi: 10.1038/ncomms8777 (2015)

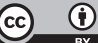

This work is licensed under a Creative Commons Attribution 4.0 International License. The images or other third party material in this article are included in the article's Creative Commons license, unless indicated otherwise in the credit line; if the material is not included under the Creative Commons license, users will need to obtain permission from the license holder to reproduce the material. To view a copy of this license, visit http://creativecommons.org/licenses/by/4.0/ 